\title{
Multi-sensor image registration by combination of Relaxation Optimization matching and Partitioning RANSAC
}

\author{
WU Yingdan ${ }^{1, a}$, MING Yang ${ }^{2, b}$ \\ ${ }^{1}$ College of Science, Hubei University of Technology, Wuhan, China \\ ${ }^{2}$ Geospatial Information and Digital Transportation Research Institute, CCCC Second Highway \\ Consultants Co., Ltd, Wuhan, China \\ ayd_wu2010@163.com, bankelbreaker@163.com
}

Keywords: multi-sensor; image registration; relaxation optimization; RANSAC

\begin{abstract}
This paper presents a multi-sensor image registration method by combination of relaxation optimization matching and Partitioning RANSAC. Firstly, the global coarse registration is performed to establish the approximate relationship of reference and slave image. Secondly, in each pyramid level, the normalized MI and relaxation optimization technique are adopted to get the matching points, and partitioning RANSAC is used to delete the existing false matches. The coarse-to-fine strategy is integrated to refine the results, and finally the rubber sheeting method is used to realize the image registration. Two datasets have been experimented, and it can be found that satisfactory registration method can be obtained.
\end{abstract}

\section{Introduction}

Image registration has a wide range of applications, such as image fusion, environment monitoring, change detection, image mosaicking, medical image processing, and map updating. Multi-sensor image registration, whose purpose is to geometrically align images of the same scene taken by different sensors or modalities. However, due to the complexity and unknown relationship between intensity of corresponding pixels, this has been a challenging problem for a long time ${ }^{[1-2]}$.

Global registration techniques are the widely used methods ${ }^{[3-4]}$.This kind of methods has gained great success in the medical image registration ${ }^{[5]}$, but their high computational complexity is the main obstacle for a wider application. For image registration of large volume, the methods mainly fall back on the local techniques, which can be roughly classified into two categories of feature based and intensity based techniques. Feature based approaches frequently use salient features such edges and points ${ }^{[6]}$, which demands that the features should be distinct, spread throughout the image, and efficiently detectable in both images. In practice, however, same features are hard to extract especially for multi-sensor images.

Therefore, a local intensity-based registration technique is proposed in this paper. The matching algorithm integrates the intensity and spatial similarity to derive the corresponding points, and coarse-to-fine strategy as well as partitioning RANSAC robust estimation algorithm is adopted to enhance matching results' reliability. Experiments have been carried out and the multi-sensor image registration results are analyzed.

\section{Proposed algorithm}

Global Coarse Registration. To robustly obtain approximate geometrical relationship between reference image and slave image, the global coarse registration is adopted. And to alleviate the heavy calculation burden, it is carried out in the highest level of pyramid level.

Global coarse registration is the process of finding approximate parameters $\boldsymbol{p}$ that minimize the objective function $\boldsymbol{F}$, namely

$$
\hat{\mathbf{p}}=\arg \min _{\mathbf{p}} F(R, S ; \mathbf{p})
$$

Here the objective function $\boldsymbol{F}$ is the normalized mutual information (MI) of reference image and 
slave image, which will be detailed later, and $\boldsymbol{p}$ is the affine transformation parameters. The Powell's method ${ }^{[7]}$ is used to perform the optimization of the objective function.

Feature Points Matching. MI describes a statistical dependence between two random variables (e.g., $A$ and $B$ ) expressed in terms of variable entropies ${ }^{[8]}$, which is defined as

$$
\operatorname{MI}(A, B)=H(A)+H(B)-H(A, B)
$$

Where $H(A)$ and $H(B)$ are the Shannon entropies of $A$ and $B$ respectively, and $H(A, B)$ is the joint variable entropy of $A$ and $B$. To reduce the sensitivity of MI toward changes in image overlap, the normalized $\mathrm{MI}^{[9]}$ is employed in this paper:

$$
\mathrm{NMI}(\mathrm{A}, \mathrm{B})=(\mathrm{H}(\mathrm{A})+\mathrm{H}(\mathrm{B})) / \mathrm{H}(\mathrm{A}, \mathrm{B})
$$

Due to the ill-posed characteristics of image matching, let alone the images from different sensors or modalities, although we can limit the search space into a small range, it is inevitable to encounter the problem of false matching and multiple matching peaks. So the normalize MI is used to find the candidate matches, and then the relaxation optimization matching is adopted to find the most correct matching points from the candidates.

Relaxation optimization treats the image matching as a labeling problem ${ }^{[10]}$. The main distinction from the single point matching methods is spatial compatibility consideration, which is realized by defining a compatible coefficient function and some constraint scheme. In most cases, the smoothness constraint is used, assuming that the terrain surface varies smoothly over the area. The details of realizing the relaxation optimization matching can be found in literature [11].

Partitioning RANSAC for outlier elimination. To ensure the robustness of matching results in each pyramid level, it is crucial to find an efficient way to detect and eliminate the false matching points. The typical way is to use two-order polynomials to depict the geometrical relationship between points in the reference image and corresponding ones in the slave image. But the good results are often obtained in the case of small image.

RANSAC is a powerful and robust estimator in the presence of outliers ${ }^{[12]}$. It does not require prior assumption of the distribution of outliers. It works by estimating a model with the minimum required number of control points selected randomly and checking whether other control points support the model. It repeats these procedures for a certain number of times and chooses the best model that has the largest supports. After that, it re-estimates the model using all supporting control points.

However, for the remote sensing imagery, the image size is usually very large, whose width and height may be several thousands of pixels. Only using the two-order polynomials is not enough to model the relationship of corresponding points.

The main idea of partitioning RANSAC method is firstly partitioning the image into several blocks, and then for each block the RANSAC algorithm with two-order polynomials is used to eliminate the false matching points.

\section{Experiment Results and Analysis}

To verify the effectiveness of the algorithm proposed in this paper, two datasets have been tested. One dataset consists of two images both from Envisat SAR sensor, and the other dataset is composed by two multi-sensor images, one is COSMO-SkyMed Level 1C product (also indicated as Geocoded Ellipsoid Corrected (GEC)) and one is IRS-P5 (also known as CARTOSAT-1) image, and image resolution are both $2.5 \mathrm{~m}$. The test images have been clipped into $5000 \times 5000$ pixels. And in the procedure of image preprocessing, all the images have been rescaled to 255 gray level, and histogram equalization is performed to make the image appearing similar as much as possible.

For dataset one, 153 pairs of corresponding points are successfully obtained, and for dataset two, 74 pairs are acquired, and the feature points are evenly distributed in the overlap area, as shown in Fig. 1. In the experiment, the registration error tolerance is set to 2 pixels of corresponding level's pyramid image, when performing partitioning RANSAC, each feature point's 
registration error can be calculated by the estimated model parameters, and the point will be regarded as false match when the error is larger than the threshold.

From Fig. 2, it can be found that the common linear feature, such as the road, the building and lake boundary, are congruent with each other accurately, and the distortion between the two images are correctly represented, and the registration results seems very satisfactory.
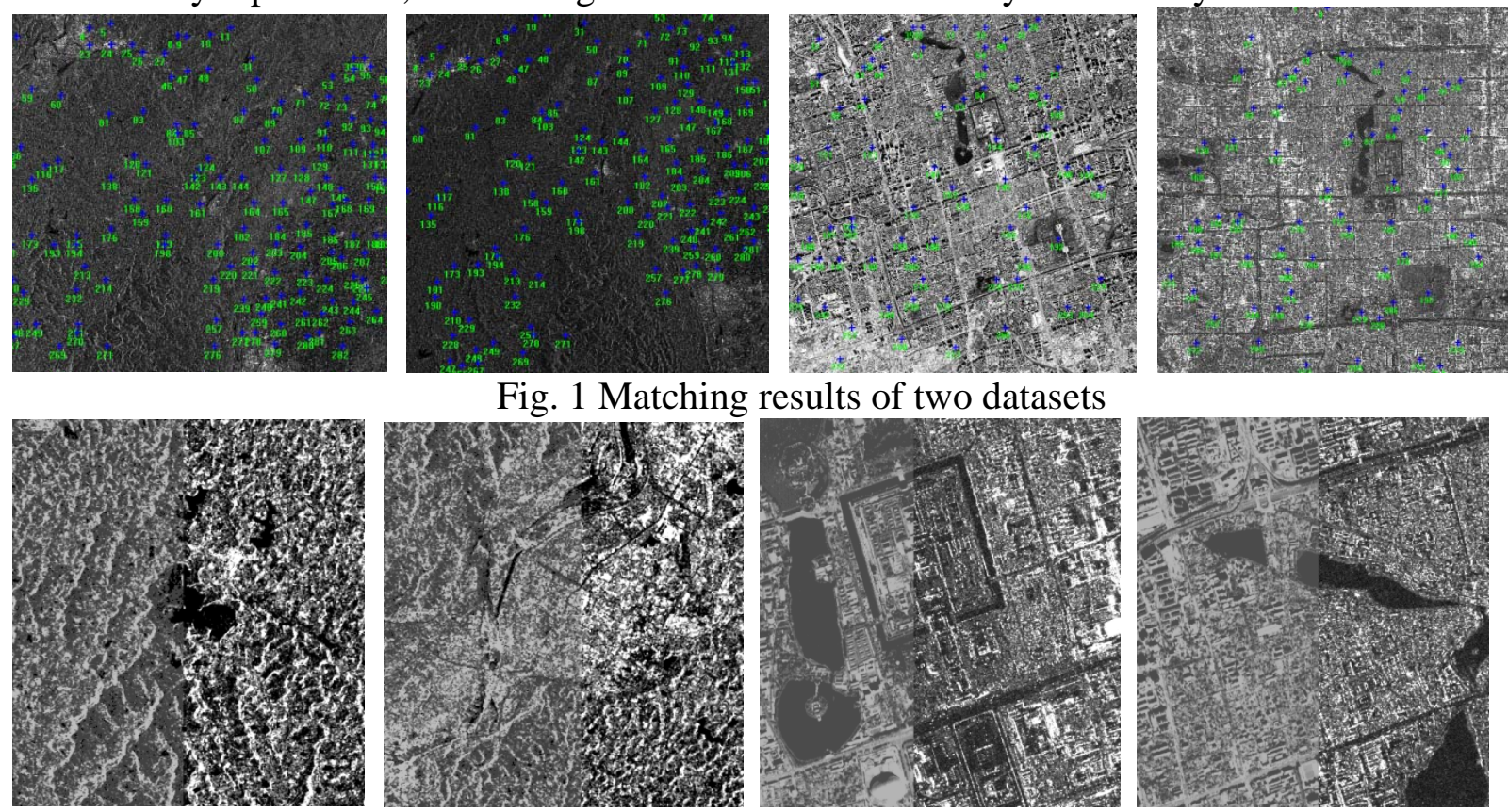

Fig. 2 The registration result of two datasets

\section{Conclusions}

This paper presents a robust registration method with pixel accuracy for multi-sensor remote sensing image. The normalized MI and relaxation optimization technique are adopted to get the matching points, and partitioning RANSAC can guarantee the robustness of matching results. The experiments have demonstrated that the method can obtain satisfactory result for multi-sensor image registration.

\section{Acknowledgement}

The research presented in this paper was carried out as part of project supported by the Natural Science Foundation of Hubei Province, China (Grant No. 2012FFB00602) and project by the Scientific Research Starting Foundation for Doctors, Hubei University of Technology (Grand No. BSQD12078).

[1] B. Zitova , J. Flusser, Image registration methods: A survey, Image Vis. Comput.. 21(2003) 977-1000.

[2] L. Fonseca, B. Manjunath, Registration techniques for multisensory remotely sensed imagery, Photogramm. Eng. Remote Sens.. 62(1996) 1049-1056.

[3] Y. SunKim, J. HakLee, J. BeomRa, Multi-sensor image registration based on intensity and edge orientation information, Pattern Recognition. 41 (2008) 3356-3365.

[4] F. Maes, A. Collignon, D. Vandermeulen, G. Marchal, P. Suetens, Multimodality image registration by maximization of mutual information, IEEE Trans. Med. Imaging. 16 (1997) 187--198.

[5] J. Pluim, J. Maintz, M. Viergever, Mutual-information-based registration of medical images: A survey, IEEE Trans. Med. Imag.. 22 (2003) 986-1004.

[6] C.R. Maurer Jr., R.J. Maciunas, J.M. Fitzpatrick, Registration of head CT images to physical space using a weighted combination of points and surfaces, IEEE Trans. Med. Imaging. 17 
(1998) 753-761.

[7] W.H. Press, B.P. Flannery, S.A. Teukolsky, W.T. Vetterling, Numerical recipes in C, Cambridge University Press, Cambridge, UK, 1992.

[8] P. Viola and W. M. Wells, Alignment by maximization of mutual information, Int. J. Comput. Vis.. 24 (1997) 137-154.

[9] C. Studholme, D.L.G. Hill, D.J. Hawkes, An overlap invariant entropy measure of 3D medical image alignment, Pattern Recognition. 32 (1999) 71-86.

[10] E.R. Hancock, J. Kittler, Discrete relaxation. Pattern Recognition, 23 (1990) 711-733.

[11] Y.D. Wu, Y. Ming, A robust registration method for high resolution remote sensing images, Geoinformatics 2008 and Joint Conference on GIS and Built Environment: Classification of Remote Sensing Images, 7147 (2008) 71470Y-1-71470Y-9.

[12] M.A. Fischler, R.C. Bolles, Random Sample Consensus: A Paradigm for Model Fitting with Applications to Image Analysis and Automated Cartography, Communications of the Association of Computing Machinery, 24 (1981) 381-395. 